\title{
Rol cardioprotector del carvedilol para pacientes en quimioterapia, revisión sistemática y metanálisis de estudios clínicos aleatorizados
}

\author{
Lucía Florio Legnani ${ }^{1}$, Pablo Alvarez Rocha ${ }^{1}$, Tomás Galvez-Olotegui ${ }^{2}$

\section{RESUMEN}

Objetivo: Los tratamientos oncológicos pueden causar alteraciones cardiovasculares pese a lograr tratamientos exitosos de la enfermedad. El rol del carvedilol como cardioprotector en este escenario es incierto. Se evaluó la evidencia existente respecto al rol del carvedilol para prevenir el deterioro de la función sistólica del ventrículo izquierdo en pacientes sometidos a quimioterapia

Materiales y métodos: Se trata de una revisión sistemática y metanálisis de ensayos clínicos aleatorizados. Se realizó una búsqueda en Pubmed, Embase, Cochrane, Scielo y clinicaltrials.gov; incluyendo trabajos que evaluaron y compararon la diferencia en la fracción de eyección del ventrículo izquierdo (antes y después de recibir quimioterapia) entre pacientes con y sin carvedilol. El tamaño del efecto se expresó como la diferencia estandarizada (d) y la diferencia de medias entre grupos con un intervalo de confianza del $95 \%$.

Resultados: Se identificaron 9749 manuscritos; se incluyeron 4 estudios con un total de 343 pacientes adultos, $86.9 \%$ de sexo femenino, con fracción de eyección del ventrículo izquierdo normal y sin historia previa de insuficiencia cardíaca. El grupo bajo tratamiento con carvedilol mostró una caída menor aunque sin diferencia estadísticamente significativa de la fracción de eyección del ventrículo izquierdo que el grupo control $(d=-0.501$ [ $-1.372,0.371] ; p=0.260 ;$ siendo la diferencia de la reducción de fracción de eyección del ventrículo izquierdo pre-post quimioterapia entre ambos grupos: $-0.71 \%[-1.88,0.575])$; sin embargo la fracción de eyección del ventrículo izquierdo final fue mayor en el grupo carvedilol $(d=0.361$ [ $0.146,0.575] ; p=0.001$; siendo el tamaño del efecto $=1.73 \%[0.74,2.72]$.

Conclusiones: El carvedilol solo o combinado, se asocia a una mayor fracción de eyección del ventrículo izquierdo, pero no a un menor descenso de la misma al finalizar la quimioterapia. Son necesarios estudios clínicos aleatorizados con mayor población, seguimiento y calidad para determinar la implicancia clínica del uso de carvedilol en pacientes sometidos a quimioterapia.

Palabras claves: Antagonistas beta adrenérgicos; quimioterapia; cardiotoxicidad (Fuente: DeCS BIREME).

\section{Cardioprotective role of carvedilol in patients on chemotherapy: systematic review and meta-analysis of randomized clinical trials}

\section{ABSTRACT}

Objective: Cancer therapy may cause cardiovascular toxicity even after successful treatment. It is unknown the cardioprotective role of carvedilol in this setting. We evaluated the role of carvedilol for the prevention of left ventricular systolic dysfunction in chemotherapy patients.

Materials and methods: A systematic review and meta-analysis of randomized clinical trials were performed through PubMed, Embase, Cochrane, SciELO and ClinicalTrials.gov, including trials that evaluated and compared the difference of left ventricular ejection fraction (pre- and post-chemotherapy) in patients with and without carvedilol treatment. The effect size was expressed as the standardized difference (d) and the mean difference between groups with $95 \%$ confidence interval.

Results: Nine thousand seven hundred forty-nine $(9,749)$ manuscripts were screened. Four $(4)$ studies consisting of a total of 343 adult patients, from which $86.9 \%$ were females with normal left ventricular ejection fraction and no past history of heart failure, were included. The carvedilol group showed less -though not statistically significant- reduction of the left ventricular ejection fraction than the control group $(d=-0.501[-1.372,0.371] ; p=0.260)$. The difference of the reduction in the left ventricular ejection fraction pre- and post-chemotherapy in both groups was $-0.71 \%[-1.88,0.46]$. However, left ventricular ejection fraction post-chemotherapy was higher in the carvedilol group $(d=0.361[0.146,0.575] ; p=0.006)$ and the effect size was $1.73 \%[0.74,2.72]$.

Conclusions: Monotherapy or combination therapy with carvedilol is associated with a higher left ventricular ejection fraction, but not with less reduction in the left ventricular ejection fraction post-chemotherapy. Long-term, larger and high-quality randomized clinical trials are required to determine the clinical implication of the use of carvedilol in patients under chemotherapy.

Keywords: Adrenergic beta-antagonists; drug therapy; cardiotoxicity (Source: MeSH NLM).

1. Universidad de la República. Montevideo, Uruguay.

2. Universidad de San Martín de Porres, Facultad de Medicina Humana. Lima, Perú. 


\section{INTRODUCCIÓN}

El cáncer es la principal causa de muerte en los países desarrollados y la segunda en los países en desarrollo ${ }^{(1)}$. El pronóstico de los pacientes con cáncer ha mejorado significativamente debido al uso de nuevos fármacos y terapias antineoplásicas y al desarrollo de regímenes más intensos que han determinado la remisión en muchos tipos de cáncer y en otros, la transformación en una enfermedad crónica. Sin embargo, estos tratamientos están asociados con efectos secundarios relevantes, dentro de ellos, la cardiotoxicidad $^{(2)}$.

El efecto de las antraciclinas sobre la función cardíaca es bien conocido y puede determinar la presencia de una disfunción sistólica del ventrículo izquierdo irreversible, que sabemos es dosis-dependiente ${ }^{(2,3)}$.Otros fármacos utilizados en hemopatías malignas, cáncer de mama y otras enfermedades oncológicas pueden producir daño cardíaco en forma independiente de la dosis. La cardiotoxicidad puede ser aguda o crónica y producirse por diferentes mecanismos incluyendo toxicidad endotelial y daño directo sobre el miocito ${ }^{(4)}$. Es importante destacar que la cardiotoxicidad y la disfunción sistólica resultante, aún en forma asintomática, pueden comprometer la sobrevida de los pacientes oncológicos a largo plazo y limitar las opciones terapéuticas disponibles ${ }^{(5)}$. Estudios de grandes dimensiones han demostrado el rol protector de algunos betabloqueantes (principalmente carvedilol, bisoprolol, metoprolol XR y nevibolol) en la progresión de la disfunción sistólica y la dilatación ventricular, aportando beneficio clínico en términos de síntomas y mortalidad en pacientes con disfunción sistólica, tanto en miocardiopatías isquémicas como no-isquémicas ${ }^{(6,7,8,9,10,11)}$; el rol de los mismos en prevenir la aparición de novo de disfunción sistólica en pacientes que reciben quimioterapia no está bien establecido. El carvedilol es el único beta bloqueante con acción antioxidante, lo que puede explicar algún beneficio adicional en el tratamiento de pacientes con cardiotoxicidad por quimioterápicos. Pocos fármacos han demostrado prevenir el deterioro de la función sistólica del ventrículo izquierdo (VI) por quimioterápicos y el carvedilol es uno de ellos. Su uso está facilitado por su relativo bajo costo y buena tolerancia clínica. Estudios clínicos aleatorizados y metanálisis muestran el efecto benéfico en otros escenarios de disfunción sistólica diferentes a la quimioterapia (QMT) ${ }^{(12)}$. Muchas guías de práctica clínica recomiendan el uso de carvedilol en pacientes con cardio toxicidad por quimioterápicos con un descenso de la fracción de eyección del ventrículo izquierdo (FEVI) mayor al $10 \%$ y un valor absoluto de la misma menor o igual a $50 \%$ independientemente de la presencia de síntomas de insuficiencia cardíaca ${ }^{(13)}$.

La evidencia disponible respecto a la eficacia del carvedilol en prevenir la aparición de disfunción sistólica del VI en pacientes expuestos a quimioterapia con función sistólica del VI (FSVI) normal previa es escasa y el tamaño del efecto no está bien definido. Pocos ensayos de pequeñas dimensiones ${ }^{(14-21)}$, evaluaron la FSVI luego de recibir quimioterapia en pacientes tratados con carvedilol y no ha sido publicado ningún metanálisis enfocado en el efecto del carvedilol en este escenario.

El objetivo del presente trabajo fue realizar una revisión sistemática de la evidencia disponible respecto al rol del carvedilol en prevenir la aparición de disfunción sistólica del VI en pacientes bajo quimioterapia y metanálisis de los resultados en vistas a precisar el tamaño del efecto terapéutico y poder comparar resultados entre diferentes situaciones clínicas.

\section{MATERIALES Y MÉTODOS}

Se trata de una revisión sistemática y meta-análisis para investigar el efecto cardioprotector del carvedilol en pacientes bajo tratamiento quimioterápico. La pregunta planteada fue si el carvedilol (solo o asociado con otras terapias cardioprotectoras), es efectivo en prevenir el deterioro de la FSVI en pacientes que reciben quimioterapia por al menos 1 mes, comparado con placebo o ninguna terapia cardioprotectora. Se comparó el grado de deterioro de la FSVI en pacientes que recibieron quimioterapia y tratamiento preventivo con carvedilol vs aquellos sin tratamiento cardio-protector o placebo. El resultado primario fue la diferencia en la FSVI pre menos post quimioterapia. Se tomaron en cuenta como parámetros de FSVI la FEVI, fracción de acortamiento del VI y/o deformación global del VI. El resultado secundario fue la FSVI post quimioterapia. El valor de FEVI, cuando estuvo disponible, se prefirió para el análisis principal de ambos resultados.

Dos investigadores independientes realizaron una búsqueda de artículos relevantes sin filtros hasta el 15 de abril del 2016 en PubMed, EMBASE, Cochrane, Scielo y una búsqueda manual de literatura gris y referencias usadas en los artículos seleccionados; se envió un correo electrónico a los principales autores con este objetivo; se buscaron estudios sin publicar en clinicaltrials.gov. Se tomó como criterio de inclusión de los trabajos que fueran estudios clínicos aleatorizados (ECA) con un score de calidad de Jadad $\geq 3$. La población del estudio debían ser adultos o niños que iniciaban quimioterapia en ausencia de síntomas previos de insuficiencia cardíaca (IC), cardiopatía estructural o disfunción sistólica (incluyendo sólo FEVI $\geq 50$ \%). El tratamiento activo debía ser carvedilol (solo o junto a otro tratamiento cardioprotector) y la comparación debía realizarse con placebo o ningún tratamiento cardioprotector. Se desarrolló la búsqueda seleccionando artículos relevantes, excluyendo aquellos no relacionados al tema e identificando duplicados. El diagrama de flujo de los estudios recolectados se construyó siguiendo las recomendaciones PRISMA ${ }^{(22)}$. La existencia de diferencias en la selección entre investigadores fueron resueltas por consenso entre ambos investigadores y un análisis de concordancia de la selección y de la calificación de Jadad fue ejecutado. Las palabras claves de búsqueda fueron: (carvedilol OR beta blocker*) and (chemotherapy OR cardiac toxicity OR anthracycline OR trastuzumab) and (systolic dysfunction OR systolic function OR heart failure OR ejection fraction OR strain). 
La información de los estudios incluidos fue: número de sujetos por grupo, adultos/niños, tipo y dosis de quimioterapia, dosis de carvedilol, presencia de tratamiento cardioprotector adicional y su tipo, FSVI pre y postquimioterapia y la diferencia entre ambas. Para el análisis estadístico se utilizó STATA 13.

El efecto combinado fue obtenido por los modelos de efecto fijo y random, prefiriendo este último por heterogeneidad entre estudios. Los resultados se muestran en gráfica de bosque. Se realizó prueba de sensibilidad del resultado primario y secundario evaluando la diferencia de FSVI a través de otros parámetros diferentes a la FEVI sí estuvieron disponibles y del resultado primario a través de la exclusión del análisis de cada uno de los estudios incluidos. Se realizó análisis de subgrupos comparando carvedilol sólo como tratamiento activo vs carvedilol más otros tratamientos cardioprotectores y se evaluó el tamaño del efecto intra estudio según FEVI vs deformación miocárdica. Se ejecutó test de Begged y Eger para investigar sesgo de publicación. La presente publicación se realiza siguiendo las recomendaciones PRISMA ${ }^{(22,23)}$.

\section{RESULTADOS}

Un total de 9771 estudios fueron identificados a través de las bases de datos, 22 duplicados fueron retirados y 9749 estudios escrutados según el título y el resumen; fueron seleccionados 64 estudios para elegibilidad; 7 incluidos para el análisis cualitativo sumando uno que se encontró por búsqueda manual a través de seguimiento de autores y de las referencias de los 64 estudios elegidos. Finalmente, fueron analizados cuantitativamente 4 estudios con un total de 343 pacientes adultos, 86,88\% mujeres (Figura 1). Se encontraron pocos trabajos que tuvieran el carvedilol como tratamiento activo o combinado con otros cardioprotectores. Los tratamientos adicionales, cuando estuvieron presentes, fueron enalapril, candesartan y trimetazidine. Solo uno de los trabajos fue doble ciego y solo 3 de ellos reportaron el uso de placebo.

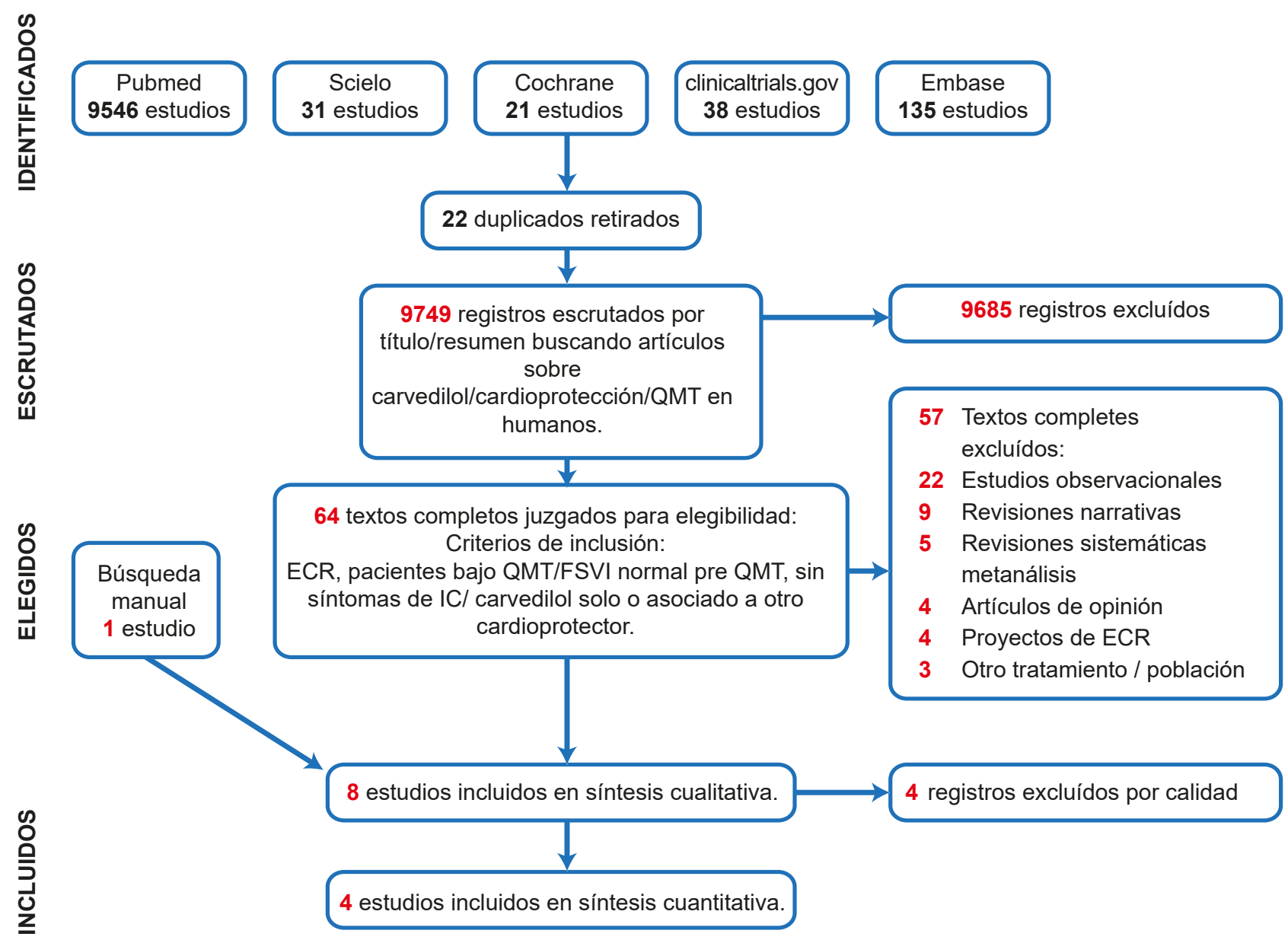

Figura 1. Diagrama de flujo de selección de estudios 
La búsqueda de estudios y la estrategia de evaluación tuvo una concordancia entre investigadores para la etapa de escrutinio de 0.6 Cl95\%(0.46-0.75) índice kappa y de 1 para la elegibilidad y la asignación del puntaje Jadad. Las características basales de los trabajos seleccionados, las variables significativas y la evaluación de sesgos se muestran en las (Tabla 1,2 y 3). Se destaca que los estudios incluidos en el análisis estadístico (Bosch 2013, Elitok 2014, Zhang 2016 and Tashakori 2016) tiene una población adulta, preponderantemente femenina en tratamiento con antraciclinas por cáncer de mama y todos sin historia previa de IC y con FEVI normal basal.

Tabla 1. Características basales de estudios seleccionados para análisis cualitativo

\begin{tabular}{|c|c|c|c|c|}
\hline $\begin{array}{c}\text { Estudio Autor, Revista, } \\
\text { Año }\end{array}$ & Población & QMT & $\begin{array}{l}\text { Tratamiento } \\
\text { Activo / control }\end{array}$ & $\begin{array}{l}\text { Seguimiento } \\
\text { (meses) }\end{array}$ \\
\hline $\begin{array}{l}\text { Kalay, } \\
\text { JACC, } \\
2006\end{array}$ & $\begin{array}{l}\text { Cualquier enfermedad } \\
\text { oncológica/adultos }\end{array}$ & ANT & Carvedilol 25 mg/ Placebo & 6 \\
\hline $\begin{array}{l}\text { Salehi, } \\
\text { Am Heart Hosp J, } \\
2011\end{array}$ & $\begin{array}{l}\text { Cáncer de mama-linfoma/ } \\
\text { adultos }\end{array}$ & ANT & $\begin{array}{c}\text { Carvedilol12.5-25mg / } \\
\text { Placebo }\end{array}$ & 4 \\
\hline $\begin{array}{l}\text { El-Shitany, } \\
\text { J Card Fail, } \\
2012\end{array}$ & Leucemia aguda/ niños & ANT & $\begin{array}{l}\text { Carvedilol } 12.5 \mathrm{mg} / \mathrm{no} \\
\text { cardioprotector }\end{array}$ & 1 \\
\hline $\begin{array}{l}\text { Bosch } \\
\text { JACC } \\
2013\end{array}$ & $\begin{array}{l}\text { Hemopatías malignas/ } \\
\text { adultos }\end{array}$ & ANT $^{*}$ & $\begin{array}{l}\text { Carvedilol } 25-50 \mathrm{mg}+ \\
\text { Enalapril } 10 \mathrm{mg} / \mathrm{no} \\
\text { cardioprotector }\end{array}$ & 6 \\
\hline $\begin{array}{l}\text { Liu } \\
\text { Ch Oncology J } \\
2013\end{array}$ & $\begin{array}{l}\text { Cáncer de mama/ adultos, } \\
\text { mujeres }\end{array}$ & ANT & $\begin{array}{c}\text { Carvedilol } 10 \mathrm{mg}+ \\
\text { Candesartan } 5 \mathrm{mg} / \mathrm{no} \\
\text { cardioprotector }\end{array}$ & 6 \\
\hline $\begin{array}{l}\text { Elitok } \\
\text { Cardiol J } \\
2014\end{array}$ & $\begin{array}{c}\text { Cáncer de mama/ adultos, } \\
\text { mujeres }\end{array}$ & ANT & $\begin{array}{l}\text { Carvedilol } 12.5 \mathrm{mg} / \mathrm{no} \\
\text { cardioprotector }\end{array}$ & 6 \\
\hline $\begin{array}{l}\text { Tashakori } \\
\text { Cardiology } \\
2016\end{array}$ & $\begin{array}{c}\text { Cáncer de mama/ adultos, } \\
\text { mujeres }\end{array}$ & ANT & Carvedilol 6.25/ Placebo & 3 \\
\hline $\begin{array}{l}\text { Zhang } \\
\text { Ch J Gen Pract } \\
2016\end{array}$ & $\begin{array}{l}\text { Cáncer de mama/ adultos, } \\
\text { mujeres }\end{array}$ & ANT & $\begin{array}{l}\text { Carvedilol } 25-50 \mathrm{mg}+ \\
\text { Trimetazidine } 60 \mathrm{mg} / \mathrm{no} \\
\text { cardioprotector }\end{array}$ & 4 \\
\hline
\end{tabular}

Tabla 2. Variables de estudios seleccionados para análisis cuantitativo

\begin{tabular}{|c|c|c|c|c|c|c|c|c|c|c|c|c|}
\hline \multirow{2}{*}{$\begin{array}{c}\text { Estudio } \\
\text { Autor, Revista, } \\
\text { Año } \\
\text { Bosch } \\
\text { JACC } \\
2013\end{array}$} & \multicolumn{2}{|c|}{$\begin{array}{c}\mathrm{N} \\
\mathrm{n} \text { activo / } \mathrm{n} \\
\text { control }\end{array}$} & \multicolumn{2}{|c|}{$\begin{array}{c}\text { FEVI } \\
\text { Pre- QMT } \\
\text { Activo/Control } \\
\text { (\%, media } \pm \text { DE) } \\
\text { Activo /control }\end{array}$} & \multicolumn{2}{|c|}{$\begin{array}{c}\text { FEVI } \\
\text { Post-QMT } \\
\text { Activo/control } \\
\text { (\%, media } \pm \text { DE) } \\
\text { Activo /controll }\end{array}$} & \multicolumn{2}{|c|}{$\begin{array}{c}\text { Diferencia FEVI } \\
(\%, \text { media } \pm \mathrm{DE}) \\
\text { Activo/control }\end{array}$} & \multicolumn{2}{|c|}{$\begin{array}{l}\text { DL post QMT } \\
\text { (\%, media } \pm \text { DE) } \\
\text { Activo/ control }\end{array}$} & \multicolumn{2}{|c|}{$\begin{array}{c}\text { Diferencia de DL } \\
\text { Active/Control } \\
(\%, \text { media } \pm D E)\end{array}$} \\
\hline & 42 & 37 & $\begin{array}{l}61.7 \\
\pm 5.1\end{array}$ & $\begin{array}{l}62.6 \\
\pm 5.4\end{array}$ & $\begin{array}{l}61.5 \\
\pm 5.1\end{array}$ & $\begin{array}{r}59.24 \\
\pm 5.4\end{array}$ & $\begin{array}{r}0.17 \\
\pm 6.84\end{array}$ & $\begin{array}{r}3.28 \\
\pm 6.85\end{array}$ & - & - & - & - \\
\hline $\begin{array}{l}\text { Elitok } \\
\text { Cardiol J } \\
2014\end{array}$ & 40 & 40 & $\begin{array}{l}66.0 \\
\pm 6.1\end{array}$ & $\begin{array}{l}65.0 \\
\pm 4.5\end{array}$ & $\begin{array}{l}64.1 \\
\pm 5.1\end{array}$ & $\begin{array}{l}63.3 \\
\pm 4.8\end{array}$ & $\begin{array}{r}1.9 \\
\pm 1.3\end{array}$ & $\begin{array}{r}1.7 \\
\pm 1.0\end{array}$ & $\begin{array}{l}20.1 \\
\pm 5.3\end{array}$ & $\begin{array}{l}16.0 \\
\pm 4.3\end{array}$ & $\begin{array}{l}0.10 \\
\pm 1.0\end{array}$ & $\begin{array}{r}3.3 \\
\pm 0.9\end{array}$ \\
\hline $\begin{array}{l}\text { Tashakori } \\
\text { Cardiology } \\
2016\end{array}$ & 30 & 40 & $\begin{array}{l}61.3 \\
\pm 3.2\end{array}$ & $\begin{array}{l}59.4 \\
\pm 4.2\end{array}$ & $\begin{array}{l}61.1 \\
\pm 3.4\end{array}$ & $\begin{array}{l}59.3 \\
\pm 4.3\end{array}$ & $\begin{array}{r}0.25 \\
\pm 1.79\end{array}$ & $\begin{array}{r}0.22 \\
\pm 0.91\end{array}$ & $\begin{array}{l}18.7 \\
\pm 5.0\end{array}$ & $\begin{array}{l}18.4 \\
\pm 4.9\end{array}$ & $\begin{array}{r}0.4 \\
\pm 4.5\end{array}$ & $\begin{array}{r}2.8 \\
\pm 2.1\end{array}$ \\
\hline $\begin{array}{l}\text { Zhang } \\
\text { Ch J Gen } \\
\text { Pract } \\
2016\end{array}$ & 58 & 56 & $\begin{array}{l}65.6 \\
\pm 4.4\end{array}$ & $\begin{array}{l}65.2 \\
\pm 4.1\end{array}$ & $\begin{array}{l}60.4 \\
\pm 4.8\end{array}$ & $\begin{array}{l}60.4 \\
\pm 4.8\end{array}$ & $\begin{array}{r}3.3 \\
\pm 0.9\end{array}$ & $\begin{array}{r}4.6 \\
\pm 0.9\end{array}$ & - & - & - & - \\
\hline
\end{tabular}


Rol cardioprotector del carvedilol para pacientes en quimioterapia, revisión sistemática y metanálisis de estudios clínicos aleatorizados

Tabla 3. Evaluación de sesgos por escala de Jadad

\begin{tabular}{|c|c|c|c|c|}
\hline Estudio & Metodo Randomización & Cegado & Seguimiento & Jadad \\
\hline Kalay, JACC, 2006(13) & $\begin{array}{c}\text { Sí } \\
\text { No especifica cual }\end{array}$ & $\begin{array}{r}\text { Simple ciego } \\
\text { Placebo }\end{array}$ & $\mathrm{Si}$ & 2 \\
\hline $\begin{array}{l}\text { Salehi, Am Heart Hosp J, } \\
\text { 2011(14) }\end{array}$ & $\begin{array}{c}\text { Sí } \\
\text { No especifica }\end{array}$ & $\begin{array}{r}\text { No especifica } \\
\text { Placebo }\end{array}$ & $\mathrm{Si}$ & 2 \\
\hline $\begin{array}{l}\text { El-Shitany, J Card Fail, } \\
2012(10)\end{array}$ & $\begin{array}{c}\text { Sí } \\
\text { No especifica cual }\end{array}$ & No & No & 1 \\
\hline $\begin{array}{l}\text { Bosch JACC } \\
2013(12)\end{array}$ & $\begin{array}{c}\text { Sí } \\
\text { Randomización } \\
\text { estratificada en bloques, } \\
\text { generada centralizada } \\
\text { por software }\end{array}$ & $\begin{array}{r}\text { Open label } \\
\text { Evaluador de } \\
\text { imágenes ciego }\end{array}$ & $\mathrm{Si}$ & 3 \\
\hline $\begin{array}{l}\text { Liu Ch Oncology J } \\
\text { 2013(11) }\end{array}$ & $\begin{array}{c}\text { Sí } \\
\text { No especifica }\end{array}$ & No & $\mathrm{Si}$ & 2 \\
\hline $\begin{array}{l}\text { Elitok Cardiol J } \\
2014(15)\end{array}$ & $\begin{array}{c}\text { Sí } \\
\text { Secuencia generada por } \\
\text { software }\end{array}$ & No & $\mathrm{Si}$ & 3 \\
\hline $\begin{array}{l}\text { Tashakori Cardiology } \\
\text { 2016(16) }\end{array}$ & $\begin{array}{l}\text { Sí } \\
\begin{array}{l}\text { Randomización en } \\
\text { bloques }\end{array}\end{array}$ & $\begin{array}{r}\text { Sí Doble ciego } \\
\text { Placebo }\end{array}$ & $\mathrm{Si}$ & 5 \\
\hline $\begin{array}{l}\text { Zhang Ch J Gen Pract } \\
2016(17)\end{array}$ & $\begin{array}{l}\text { Tabla para número } \\
\text { random }\end{array}$ & No & $\mathrm{Si}$ & 3 \\
\hline
\end{tabular}

No se encontró diferencias entre grupo carvedilol y control en el resultado primario $(\mathrm{d}=-0.501 \mathrm{Cl} 95 \%-1.372-0.371 ; \mathrm{p}$ 0.260); pero la FEVI fue mejor en el grupo carvedilol tras el tratamiento con quimioterapia con una $\mathrm{d}=0.361 \mathrm{CI} 95 \% 0.146$ 0.575; p 0.001 (resultado secundario) (Figuras 2 y 3). El tamaño del efecto mostrado a través de las diferencias de medias (Tablas 4 y 5). En cuanto a las gráficas de bosque se muestran en los sectores superiores los estudios que evaluaron función sistólica con deformación longitudinal además de FEVI y que utilizaron, además, solo carvedilol como tratamiento activo (Tashakori y Elitok) y en los inferiores aquellos que asociaron otro cardioprotector y sólo evaluaron función sistólica con FEVI (Zhang y Bosch).

Tabla 4. Resultado primario mostrando el tamaño del efecto como diferencia de media. Todos los resultados expresan FEVI

\begin{tabular}{|c|c|c|c|c|c|c|c|c|}
\hline \multirow[t]{2}{*}{ Estudio } & \multicolumn{3}{|c|}{ Carvedilol } & \multicolumn{3}{|c|}{ Control } & \multirow[t]{2}{*}{ Peso } & \multirow{2}{*}{$\begin{array}{l}\text { Diferencia de media } \\
\text { Random\%,IC95\% }\end{array}$} \\
\hline & Media \% & DE \% & Total & Media \% & DE\% & Total & & \\
\hline Tashakori 2016 & 0.25 & 1.79 & 30 & 0.22 & 0.91 & 49 & $28.6 \%$ & $0.03[-0.67,0.73]$ \\
\hline Elitok 2014 & 1.90 & 1.26 & 40 & 1.70 & 0.97 & 40 & $30.1 \%$ & $0.20[-0.29,0.69]$ \\
\hline Zhang 2016 & 3.31 & 0.85 & 58 & 4.79 & 0.85 & 56 & $31.1 \%$ & $-1.48[-1.79,-1.17]$ \\
\hline Bosch 2013 & 0.17 & 6.84 & 42 & 3.28 & 6.85 & 37 & $10.2 \%$ & $-3.11[-6.14,-0.08]$ \\
\hline Total (95 \% Cl) & & 170 & & & 173 & & $100.0 \%$ & $-0.71[-1.88,0.46]$ \\
\hline
\end{tabular}


Tabla 5. Resultado secundario mostrando tamaño del efecto como diferencia de media. Todos los resultados expresan FEVI. $p=0.0005, I^{2}=0 \%$

\begin{tabular}{|c|c|c|c|c|c|c|c|c|}
\hline \multirow[t]{2}{*}{ Estudio } & \multicolumn{3}{|c|}{ Carvedilol } & \multicolumn{3}{|c|}{ Control } & \multirow[t]{2}{*}{ Peso } & \multirow{2}{*}{$\begin{array}{l}\text { Diferencia de media } \\
\text { Random \% , IC95 \% }\end{array}$} \\
\hline & Media \% & DE \% & Total & Media \% & DE \% & Total & & \\
\hline Tashakori 2016 & 61.1 & 3.4 & 30 & 59.3 & 4.3 & 49 & $29.9 \%$ & $1.80[-0.00,3.60]$ \\
\hline Elitok 2014 & 64.1 & 5.1 & 40 & 63.3 & 4.8 & 40 & $20.7 \%$ & $0.80[-1.37,2.97]$ \\
\hline Zhang 2016 & 62.4 & 4.8 & 58 & 60.4 & 4.8 & 56 & $31.4 \%$ & $2.00[0.24,3.76]$ \\
\hline Bosch 2013 & 61.5 & 5.1 & 42 & 59.3 & 5.4 & 37 & $18.0 \%$ & $2.20[-0.13,4.53]$ \\
\hline Total $(95 \% \mathrm{Cl})$ & & 170 & & & 173 & & $100.0 \%$ & $1.73[0.74,2.72]$ \\
\hline
\end{tabular}

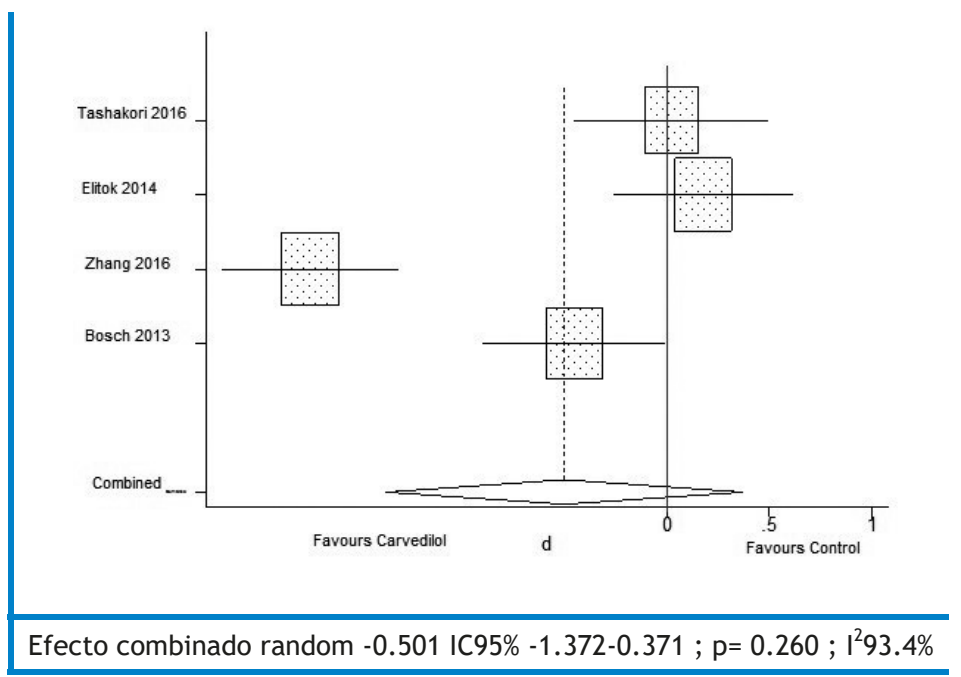

Figura 2. Resultado primario, modelo random de diferencia de FEVI pre-post QMT

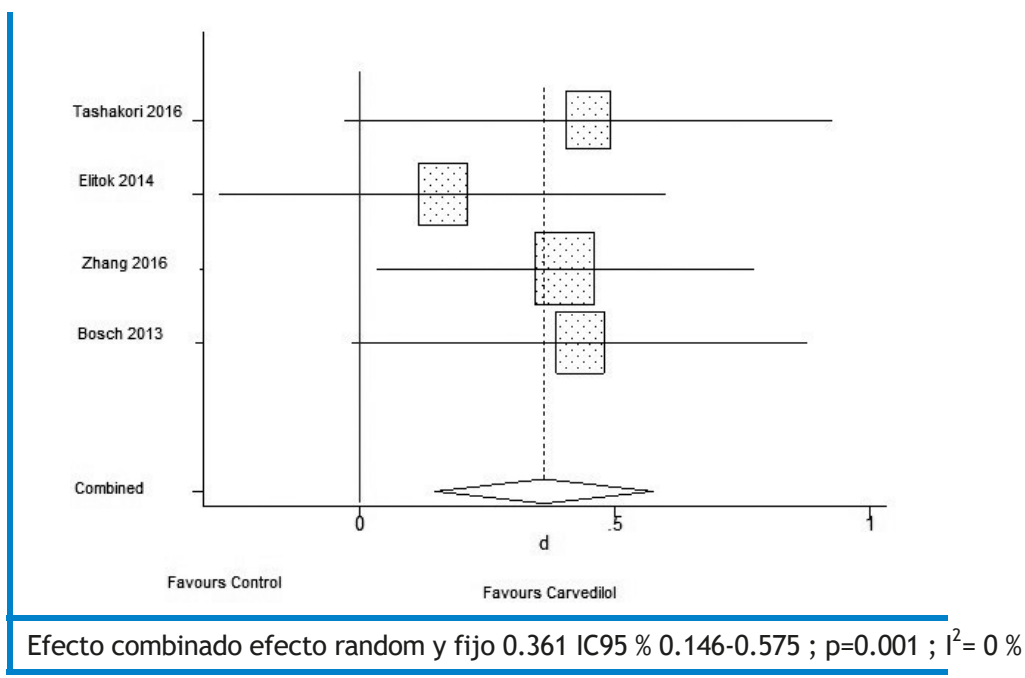

Figura 3. Resultado secundario, modelo fijo y random de FEVI post QMT 
Respecto al análisis de subgrupos, el carvedilol parece ser más efectivo cuando se utiliza combinado (Tabla 6); aunque debemos destacar que también la dosis utilizada fue de $6.25 \mathrm{mg}$ vs $25-50 \mathrm{mg}$ en los trabajos que combinaron. La evaluación de sesgos de publicación arrojó una p mayor a 0.1 en ambos casos. Se realizó análisis de sensibilidad y se desarrolló el resultado primario, incluyendo la deformación miocárdica cuando estaba disponible en vez de FEVI ; lo que encontramos es un resultado significativo que favorece al carvedilol (Figura 4); en cuanto al resultado secundario el mismo permanece significativo y favoreciendo al grupo carvedilol (Figura 5). El análisis de sensibilidad para el resultado primario, omitiendo un estudio por vez, no muestra diferencias significativas en el resultado combinado salvo un mayor intervalo de confianza en la mayoría (Figura 6).

Tabla 6. Análisis de subgrupos, diferencia de medias de FEVI pre-post QMT; carvedilol como grupo activo (Tashakori y Elitok) vs carvedilol más otro cardioprotector (Bosch y Zhang).

$\begin{array}{ccccc}\text { Subgrupos } & \text { Diferencia de media } & I C 95 \% & p & I^{2} \\ \text { Carvedilol solo } & 0.14 & -0.26,0.55 & 0.48 & 0 \% \\ \begin{array}{l}\text { Carvedilol+otro } \\ \text { cardioprotector }\end{array} & -1.57 & -2.31,-0.83 & <0.0001 & 9 \%\end{array}$

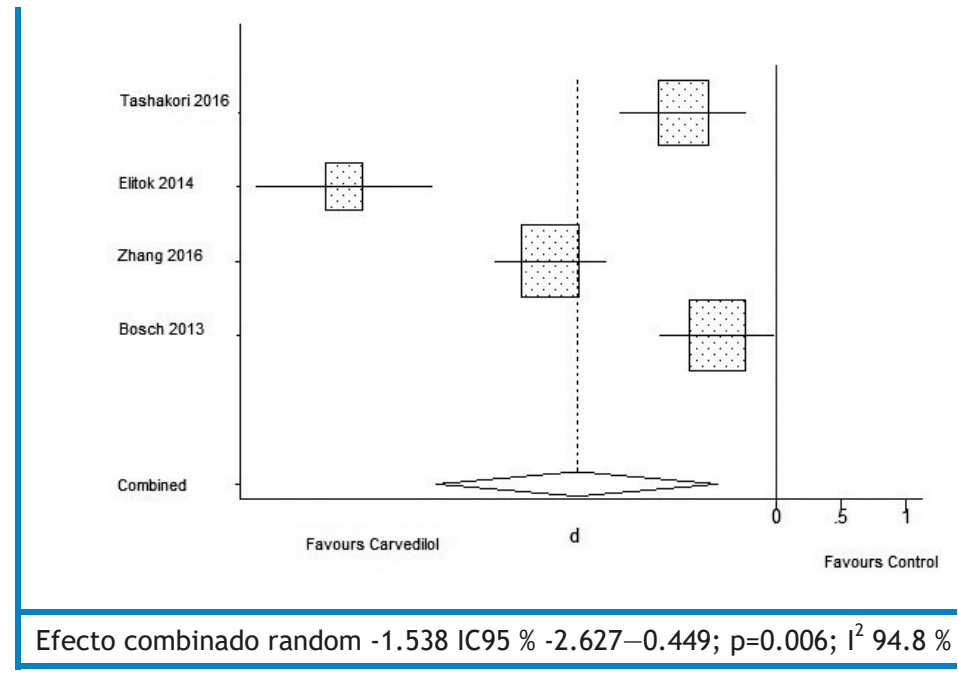

Figura 4. Análisis de sensibilidad: Gráfico de bosque de efecto random de la diferencia de FSVI (incluye DL en 2 estudios: Tashakori y Elitok) 


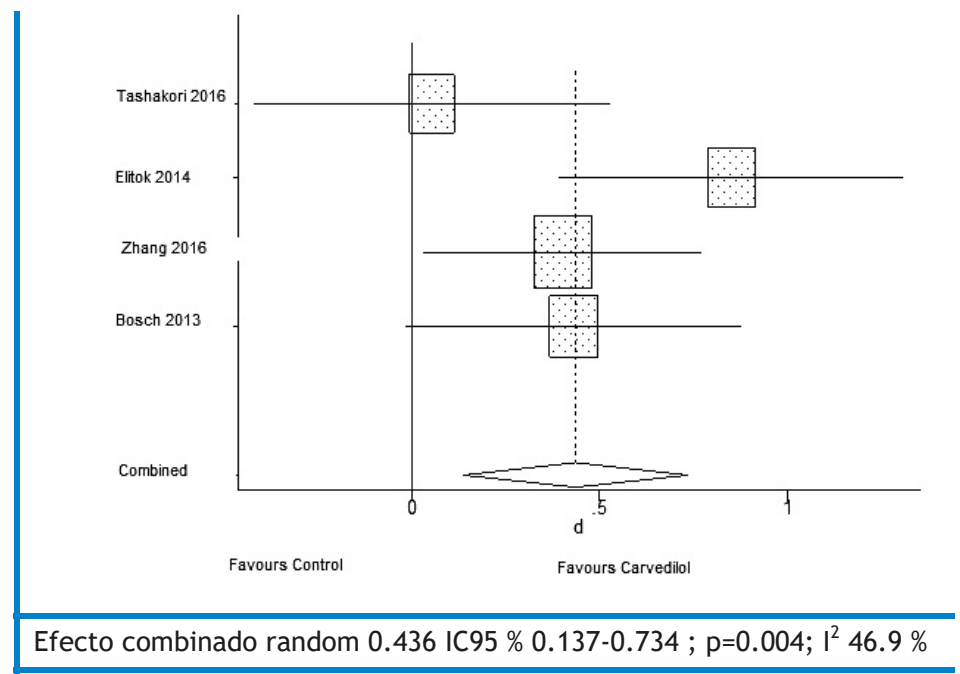

Figura 5. Análisis de sensibilidad: gráfico de bosque con efecto random de FSVI post QMT (incluye DL en 2 estudios: Tashakori y Elitok).

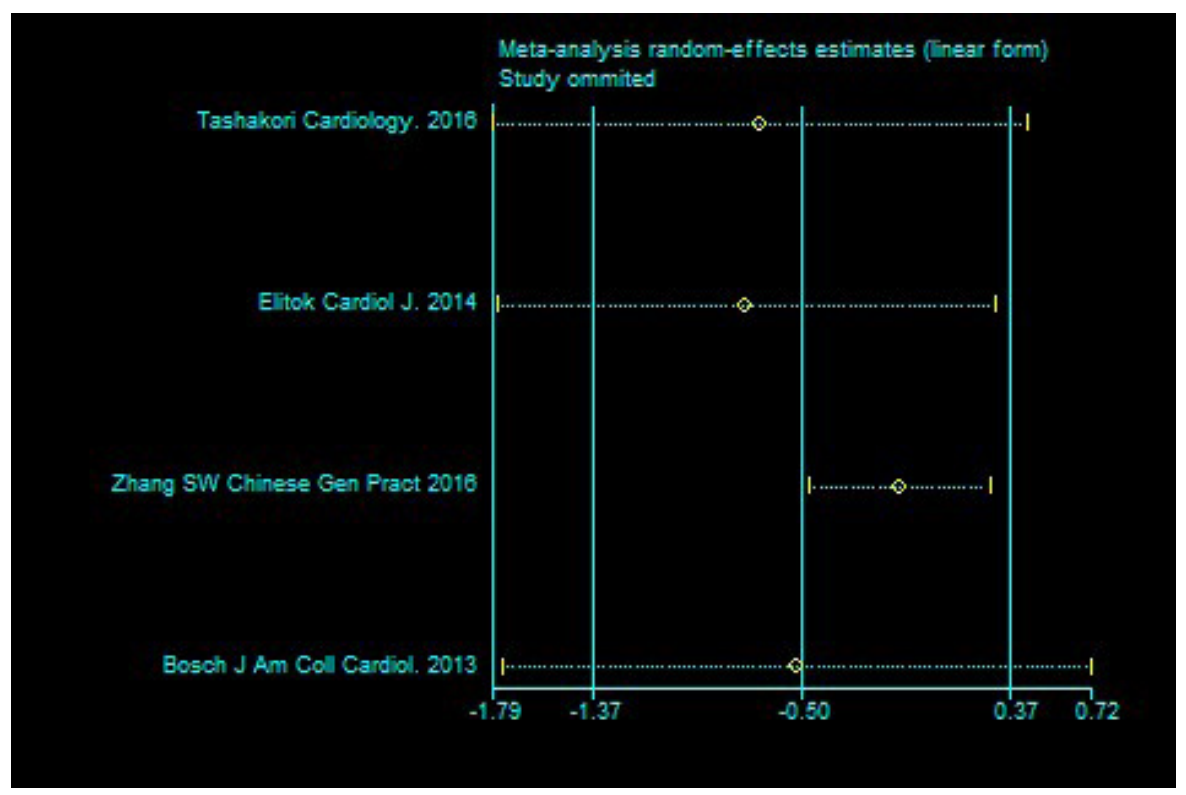

Figura 6. Análisis de sensibilidad, diferencia de FEVI pre-post QMT, omitiendo un estudio por vez

\section{DISCUSIÓN}

El presente es el primer metanálisis (MA) de ECA centrado en el efecto del carvedilol en prevenir el deterioro de la función sistólica en pacientes bajo QMT. Se trata de un pequeño MA de 4 estudios incluyendo 343 pacientes. Los resultados no son significativos para demostrar el menor deterioro de la FEVI en aquellos tratados con carvedilol, si bien tras la QMT el grupo activo tiene una FEVI significativamente más alta que el grupo control.
Este estudio refleja la preocupación de la comunidad médica en la prevención, más que en el tratamiento del deterioro de la FSVI tras el inicio de QMT ${ }^{(24)}$. Aunque hay poca evidencia respecto a este tema, MA previos que evaluaron a los beta bloqueantes y antagonistas de la angiotensina en pacientes tratados con QMT habían mostrado una mejor FEVI final en el grupo activo ${ }^{(25)}$, y un MA reciente de ECR que incluyó 5 estudios y 317 pacientes, testeando betabloqueantes como grupo en el escenario de QMT, mostró una disminución significativa de la FEVI en el grupo control y una disminución significativa de la mortalidad por todas las causas en el 
grupo betabloqueantes ${ }^{(26)}$. Pensamos que nuestro trabajo con un bajo número de pacientes incluidos, que además son de bajo riesgo de mortalidad a corto plazo (mayoría mujeres con cáncer de mama) y con breve seguimiento no tiene el poder para evaluar resultados clínicos.

Nuestro MA, así como otros respecto a este tema, muestra gran heterogeneidad de resultados entre estudios. Este hecho se explica porque se incluyeron diferentes enfermedades oncológicas, con diferentes terapias y dosis (aunque las antraciclinas son testeadas en la mayoría de los estudios), y diferentes tratamientos activos efectivizados. Nuestros resultados muestran alta heterogeneidad respecto al resultado primario pero, llamativamente, ninguna heterogeneidad al analizar el resultado secundario. Esto resulta interesante pues nuestro $M A$ incluye una población bastante homogénea de mujeres adultas con cáncer de mama, tratada con antraciclinas y que recibieron, como cardioprotector, carvedilol sólo o asociado en el grupo activo; además la FEVI es el método de evaluación de la FSVI más ampliamente utilizado y validado con una baja variabilidad intra e interobservador. Sin embargo no encontramos una explicación plausible de la diferencia en la heterogeneidad entre el resultado primario y secundario. El análisis de subgrupos muestra un efecto mejor cuando el carvedilol es combinado con otro agente cardioprotector. Esto no sorprende pues en el escenario de la IC, aquellos tratamientos que han demostrado ser clínicamente efectivos (betabloqueantes, inhibidores de la enzima de conversión de angiotensina y antagonistas de la aldosterona) se potencian en el beneficio clínico. La trimetazidina no es un tratamiento probado para la IC. La trimetazidina tiene un efecto metabólico antioxidante que puede ser útil en los pacientes bajo los efectos de QMT y que puede ser sinérgico con el papel antioxidante conocido del carvedilol ${ }^{(13)}$. La trimetazidina ha sido más usada para el tratamiento del angor en la cardiopatía isquémica, sin embargo pequeños estudios han demostrado también una mejoría de la FSVI en la cardiopatía isquémica ${ }^{(27,28,29)}$. Es destacable por su relevancia, que la dosis de carvedilol utilizada en los estudios que testearon carvedilol solo y que también evaluaron la FSVI por DL fue mucho menor que en los estudios que combinaron el carvedilol y optaron por dosis estándar del mismo. Por lo tanto, la mayor dimensión del efecto se puede deber tanto a la combinación de cardioprotectores, como al uso de una dosis óptima de cardioprotección del carvedilol.

Aunque significativa, la magnitud del efecto respecto al resultado secundario es modesta, con una diferencia de FEVI entre grupo activo/control de $1.73 \%$; sin embargo, la relevancia clínica de este efecto no es despreciable ya que la FEVI es el marcador pronóstico más destacable en la IC y en la cardiología en general ${ }^{(30)}$. Es interesante recordar que los pacientes que reciben QMT se encuentran en un estadio A de IC con un riesgo de desarrollar síntomas de IC más alto que la población general y que la identificación de disfunción sistólica asintomática posiciona al paciente en un estadio $\mathrm{B}^{(31)}$. La IC tiene un pronóstico vital a largo plazo peor que la mayoría de los cánceres y en particular respecto a la población de nuestro $M A$, la IC tiene mayor mortalidad que el cáncer de mama ${ }^{(32)}$, por eso se deben realizar grandes esfuerzos para evitar una segunda patología con peor pronóstico, en aquellas mujeres con cáncer de mama que reciben QMT, Esta justificación es suficiente a favor del uso de carvedilol como cardioprotector, más aún si consideramos sus leves( y fáciles de controlar) efectos secundarios (bradicardia, hipotensión, broncoespasmo) y su bajo costo. El MA de Manrique ${ }^{(26)}$ que testeó betabloqueantes en general, encontró un efecto mayor pero aún modesto (FEVI 5.17 \% mayor en el grupo activo). Es interesante analizar este resultado por subgrupos pues el efecto de los betabloqueantes no es comparable como grupo, ya que algunos como el atenolol no ha demostrado efecto cardioprotector en el escenario de la enfermedad oncológica $^{(33)}$.

Tashakori et al. ${ }^{(20)}$ relacionaron el efecto cardioprotector del carvedilol contra las antraciclinas no con su efecto B-bloqueante sino con el efecto antioxidante y la supresión de la producción de radicales libres de oxígeno a través de la inhibición de la nicotinamida dinucleotido deshidroginasa exógena (NADH-D); este efecto podría ser potenciado por la trimetazidina según los resultados de Zhang et al. ${ }^{(21)}$.

Respecto a los diferentes modos de evaluar la FSVI, ya fue mencionado, la FEVI es el parámetro ecocardiográfico más utilizado tanto en la práctica clínica como en los grandes ensayos clínicos. Sin embargo, la FEVI es altamente dependiente de las condiciones de carga. Estudios que han utilizado nuevos parámetros ecocardiográficos para evaluar la FSVI como la deformación miocárdica global o la velocidad de la deformación miocárdica han demostrado que la deformación longitudinal global es un buen predictor precoz de disfunción sistólica ${ }^{(34,35)}$; Stoodley et al. ${ }^{(36)}$ reportaron un $10 \%$ de reducción de la DL en la mitad de los pacientes que recibieron antraciclinas y que no tenían disminución previa de la FEVI. La QMT es el escenario clínico en el cual el uso de la DL está más validado y recomendado para el monitoreo de la FSVI. Parece lógico que tener en cuenta un parámetro más sensible como la DL aumente la capacidad de detectar diferencias entre grupos a través de un mayor tamaño del efecto tal como ocurrió en nuestro MA cuando comparamos DL con FEVI.

Nuestro estudio presentó algunas limitaciones como el bajo número de estudios y pacientes incluidos; con algunos estudios en fase 2 lo que explica el bajo número de pacientes y también el resultado principal seleccionado. Los resultados primario y secundario, aunque íntimamente relacionados con los resultados clínicos son resultados subrogantes. El análisis de estudios debe ser considerado con cautela pues solo dos grupos fueron incluidos en cada rama. Se encontró alta heterogeneidad que ha sido explicada parcialmente. Todos los pacientes incluidos recibieron antraciclinas, no hay datos de pacientes tratados con trastuzumab o similares.

En conclusión, el carvedilol se asocia a menor deterioro de la FSVI en pacientes que reciben tratamiento con antraciclinas, aunque no mostró significación estadística. Este efecto es mayor y significativo cuando se asocia con otros tratamientos cardioprotectores y/o se usa el 
carvedilol en dosis estándar. La terapia preventiva con carvedilol se asocia a mayor FEVI al finalizar el tratamiento con antraciclinas.

\section{RECOMENDACIONES}

Los resultados del presente meta-análisis justifican futura investigación en el tema. Se necesitan más y mejores ECA para definir el efecto del carvedilol sobre la función sistólica y otros resultados clínicos en el escenario de la cardiotoxicidad por quimioterápicos, que puedan tener implicancias en la práctica médica.

\section{REFERENCIAS BIBLIOGRÁFICAS}

1- World Health Organization. The Global Burden of Disease: 2004 Update. Geneva: World Health Organization; 2008. Disponible en: http://www.who.int/healthinfo/global_ burden_disease/2004_report_update/en/

2- Curigliano G, Cardinale D, Suter T, Plataniotis G, de Azambuja E, Sandri M, Goldhirsch A, Cipola C, Roila F. Cardiovascular toxicity by chemotherapy: targeted agents and radiotherapy: ESMO Clinical Pactice guidelines. Annals of Oncology. 2012; 23 (7):155-166. Disponible en: https://www.ncbi.nlm.nih.gov/ pubmed/22997448

3- Suter TM, Ewer MS. Cancer drugs and the heart: Importance and management. Eur Heart J. 2013;34(15):1102-1111. Disponible en: https://www.ncbi.nlm.nih.gov/pubmed/22789916

4- Romond EH, Perez EA, Bryant J et al. Trastuzumab plus adjuvant chemotherapy for operable HER2-positive breast cancer. N Engl J Med 2005; 353(16):1673-1684. Disponible en: https: / / www.ncbi.nlm.nih.gov/pubmed/16236738

5- Lipshultz SE, Sallan SE. Cardiovascular abnormalities in long-term survivors of childhood malignancy.J Clin Oncol. 1993;11:1199-1203. Disponible en: http://ascopubs.org/doi/ abs/10.1200/jco.1993.11.7.1199?journalCode=jco

6- Group M-HS. Effect of metoprolol CR/XLin chronic heart failure: metoprolol CR/XL randomised intervention trial in-congestive heart failure (MERIT-HF). The Lancet. 1999;353(9169):20017. Disponible en: http://www.sciencedirect.com/science/ article/pii/S0140673699044402

7- CIBIS II Investigators and Committees. The Cardiac Insufficiency Bisoprolol Study (CIBIS II): a randomized trial. Lancet. 1999; 353(9146):9-13. Disponible en: http://www. sciencedirect.com/science/article/pii/S0140673698111819

8- Packer M, Fowler MB, Roecker EB, Coats AJ, Katus HA, Krum $\mathrm{H}$, et al. Effect of carvedilol on the morbidity of patients with severe chronic heart failure. Circulation. 2002;106(17):2194-9. Disponible en: http://circ.ahajournals. org/content/106/17/2194.short

9- Packer M, Bristow MR, Cohn JN, Colucci WS, Fowler MB, Gilbert EM, et al. The effect of carvedilol on morbidity and mortality in patients with chronic heart failure. N Engl J Med. 1996;334(21):1349-55. Disponible en: http://www.nejm.org/ doi/full/10.1056/NEJM199605233342101\#t=article

10- Australia/New Zealand Heart Failure Research Collaborative Group. Randomized placebo-controlled trial of carvedilol in patients with congestive heart failure due to ischemic heart disease. Lancet 1997;349(9049):375.

11- Heidenreich P, Lee T, Massie B. Effect of beta blockade on mortality in patients with heart failure: a meta-analysis of randomized clinical trials. JACC 1997; 30(1):27-34. Disponible en: http://www.sciencedirect.com/science/article/pii/ S0735109797001046

12- Wali R, lyengar M, Beck G, Chartyan D, Chonchol M, Lukas M, Cooper C, Himmelfarb J, Weir M, Berl T, Henrich W, Cheung A.
Efficacy and Safety of Carvedilol in Treatment of Heart Failure with Chronic Kidney Disease: A Meta-Analysis of Randomized Trials Circ Heart Fail. 2011;4:18-26.

13- Bovelli D, Plataniotis G, Roila F. Cardiotoxicity of therapeuthics agents and radiotherapy related heart disease; \}: ESMO Clinical Practice Guidelines. Annals of Oncology 2010; 21(5):v27782. Disponible en: https://academic.oup.com/announc/ article21/suppl_5/v277/194029

14- El-Shitany NA, Tolba OA, El-Shanshory MR, El-Hawary EE. Protective effect of carvedilol on Adriamycin left-induced left ventricular dysfunction in children with acute lymphoblastic leukemia. J Card Fail . 2012;18(8):607-13. Disponible en:http://www.sciencedirect.com/science/article/pii/ S1071916412006124

15- Liu L, Liu Z, Liu Y, Zheng Z, Liang X, Han Y, et al. Preventive effect of low dose carvedilol combined with candesartan on the cardiotoxicity of anthracyline drugs in the adjuvant chemotherapy of breast cancer. Zhongua Zhong Liu Za Zhi. 2013;35(12):936-40. Disponible en: http://europepmc.org/ abstract/med/24506965

16- Bosch X, Rovira $M$, Sitges $M$, Domènech $A$, Ortiz-Pérez JT, de Caralt TM, et al. Enalapril and carvedilol for preventing chemotherapy induced left ventricular systolic dysfunction in patients with malignant hemopathies. JACC. 2013;61(23):235562. Disponible en: http://www.sciencedirect.com/science/ article/pii/S0735109713013983

17- Kalay N, Basar E, Ozdogru I, Er O, Cetinkaya Y, Dogan A, et al. Protective effects of carvedilol against anthracycline-induced cardiomyopathy. JACC. 2006;48(11):2258-62. Disponible en: http://www.onlinejacc.org/content/48/11/2258

18- Salehi R, Zamani B, Esfehani A, Ghafari S, Abasnezhad M, Goldust M. Protective effect of carvedilol in cardiomyopathy caused by anthracyclines in patients suffering from breast cancer and lymphoma. The Am Heart Hosp J. 2011;9(2):958. Disponible en: http://europepmc.org/abstract/ med $/ 24839645$

19- Elitok A, Oz F, Cizgici AY, Kilic L, Ciftci R, Sen F, et al. Effect of carvedilol on silent anthracycline-induced cardiotoxicity assessed by strain imaging: a prospective randomized controlled study with six-month follow-up. Cardiology journal. 2014;21(5):509-15. Disponible en: https://journals. viamedica.pl/cardiology journal/article/view/35557

20- Beheshti AT, Toroghi HM, Hosseini G, Zarifian A, Shandiz $\mathrm{FH}$, Fazlinezhad A. Carvedilol administration can prevent doxorubicin-induced cardiotoxicity: a double-blind randomized trial. Cardiology. 2016;134(1):47-53. Disponible en: https://www.karger.com/Article/PDF/442722

21- Zhang SW, Zhang XX, Shi ZC, et al. Heart protective effect of carvedilol combined with trimetazidine on breast cancer patients receiving anthracycline. Chinese General Practice. 2016; (19)7:794-798

22- Hutton B, Salanti G, Caldwell DM, Chaimani A, Schmid CH, Cameron C, et al. The PRISMA Extension Statement for Reporting of Systematic Reviews Incorporating Network Meta-analyses of Health Care Interventions: Checklist and ExplanationsPRISMA Extension for Network Meta-analysis. Ann Intern Med. 2015;162(11):777-84.Disponible en: http:// annals.org/aim/fullarticle/2299856/prisma-extensionstatement-reporting-systematic-reviews-incorporatingnetwork-meta-analyses

23- Moher D, Liberati A, Tetzlaff J, Altman DG, Group P. Preferred reporting items for systematic reviews and meta-analyses: the PRISMA statement. PLoS medicine. 2009;6(7):e1000097. Disponible en: https://www.ncbi.nlm. nih.gov/pubmed/19622511

24- Huang C, Zhang X, Ramil JM, Rikka S, Kim L, Lee Y, et al. Juvenile exposure to anthracyclines impairs cardiac progenitor cell function and vascularization resulting in 
greater susceptibility to stress-induced myocardial injury in adult mice. Circulation. 2010;121(5):675-83. Disponible en: http://europepmc.org/articles/pmc2834271

25- Yun S, Vincelette ND, Abraham I. Cardioprotective role of B-blockers and angiotensin antagonists in early-onset anthracyclines-induced cardiotoxicity in adult patients: a systematic review and meta-analysis. Postgrad Med J. 2015;91(1081):627-33. Disponible en: http://pmj.bmj.com/ content $/ 91 / 1081 / 627$.abstract

26- Manrique CR, Spinetto PV, Tiwari N, Romero J, Jorde U, Garcia M. Protective Effect of Beta-Blockers on Chemotherapy Induced Cardiomyopathy: a Meta-Analysis of Prospective Studies.(abstract) J Am Coll Cardiol. 2016; 67(13):1529. Disponible en: https://www.infona.pl/resource/bwmeta1. element.elsevier-c2e8492d-5b4e-3a41-9067-29e3f6ad91e1

27- Sellier P, Broustet J-P. Assessment of anti-ischemic and antianginal effect at trough plasma concentration and safety of trimetazidine MR $35 \mathrm{mg}$ in patients with stable angina pectoris. Am J Cardiovasc Drugs. 2003;3(5):361-9. Disponible en: https://link.springer.com/ article/10.2165/00129784-200303050-00007

28- Kowalski J, Banach M, Barylski M, Irzmanski R, Pawlicki L. Carvedilol modifies antioxidant status of patients with stable angina. Cellular \& molecular biology letters. 2008;13(2):230. Disponible en: https://cmbl.biomedcentral. com/articles/10.2478/s11658-007-0049-3

29- Brottier L, Barat J, Combe C, Boussens B, Bonnet J, Bricaud $\mathrm{H}$. Therapeutic value of a cardioprotective agent in patients with severe ischaemic cardiomyopathy. Eur Heart J. 1990;11(3):207-12. Disponible en: https://academic.oup. com/eurheartj/article-abstract/11/3/207/400317

30- Levy WC, Mozaffarian D, Linker DT, Sutradhar SC, Anker SD, Cropp AB, et al. The Seattle heart failure model. Circulation. 2006;113(11):1424-33.Disponible en: http://circ.ahajournals. org/content/113/11/1424. short

31- Ammar KA, Jacobsen SJ, Mahoney DW, Kors JA, Redfield MM, Burnett JC, et al. Prevalence and prognostic significance of heart failure stages. Circulation. 2007;115(12):1563 70. Disponible en: http://circ.ahajournals.org/ content/115/12/1563.short

32- Stewart S, MacIntyre K, Hole DJ, Capewell S, McMurray JJ. More 'malignant'than cancer? Five-year survival following a first admission for heart failure. Eur J Heart Fail. 2001;3(3):315-22. Disponible en: http://onlinelibrary.wiley. com/doi/10.1016/S1388-9842(00)00141-0/full

33- Machado V, Cabral A, Monteiro P, Goncalves L, Providencia
L. Carvedilol as a protector against the cardiotoxicity induced by anthracyclines (doxorubicin). Rev Port Cardiol. 2008;27(10):1277-96. Disponible en: http://europepmc.org/ abstract/med/19178029

34- Brown J, Jenkins C, Marwick TH. Use of myocardial strain to assess global left ventricular function: a comparison with cardiac magnetic resonance and 3-dimensional echocardiography. Am Heart J. 2009;157(1):102-5. Disponible en: http://www.sciencedirect.com/science/article/pii/ S000287030800820X

35- Feigenbaum H, Mastouri R, Sawada S. A practical approach to using strain echocardiography to evaluate the left ventricle. Circ J. 2012;76(7):1550-5. Disponible en: https://www. jstage.jst.go.jp/article/circj/76/7/76_CJ-12-0665/_article/char/ja/

36- Stoodley PW, Richards DA, Hui R, Boyd A, Harnett PR, Meikle $\mathrm{SR}$, et al. Two-dimensional myocardial strain imaging detects changes in left ventricular systolic function immediately after anthracycline chemotherapy. Eur J Echocardiogr. 2011;12(12):945-52. Disponible en: https://academic.oup. com/ehjcimaging/article/12/12/945/2396952

Fuentes de financiamiento:

Este artículo ha sido financiado por los autores.

Conflictos de interés:

Los autores declaran no tener ningún conflicto de interés.

\section{Correspondencia:}

Lucia Florio Legnani

Dirección: Gonzalo Ramírez 1869/1001. Montevideo, Uruguay

Teléfono: +59824871515

Correo electrónico: lu.floriol@gmail.com

Recibido: 25 de abril de 2017 Evaluado: 27 de mayo de 2017 Aprobado: 08 de junio de 2017

(c) La revista. Publicado por Universidad de San Martín de Porres, Perú. (c) в bajo términos de Licencia Creative Commons Atribución 4.0 Internacional. (http://creativecommons.org/licenses/by/4.0/)

ORCID iDs

Lucía Florio Legnani

Pablo Álvarez Rocha

Tomás Gálvez Olotegui https: / / orcid.org/0000-0002-9608-2666 https: / / orcid.org/0000-0002-4550-3483 https:// orcid.org/0000-0002-2177-2849 